\title{
EFEITO DE INCÊNDIOS FLORESTAIS NA ESTRUTURA E COMPOSIÇÃO FLORÍSTICA DE UMA ÁREA DE CERRADO SENSU STRICTO NA FAZENDA ÁGUA LIMPA-DF ${ }^{1}$
}

Nilton Cesar Fiedler ${ }^{2}$, Isaac Nuno Carvalho de Azevedo 2 , Alba Valéria Rezende ${ }^{2}$, Marcelo Brilhante de Medeiros ${ }^{3} \mathrm{e}$ Fábio Venturoili ${ }^{4}$

\begin{abstract}
RESUMO - O objetivo desta pesquisa foi avaliar o efeito do fogo na estrutura e composição florística de uma área de 10 ha de cerrado sensu stricto, localizada na fazenda Água Limpa da Universidade de Brasília. Foram realizados dois inventários, sendo o primeiro em 1999, logo após a área ter sido totalmente queimada, devido a um incêndio criminoso, e o segundo em novembro de 2002. Quatro parcelas de 0,1 ha $(20 \times 50 \mathrm{~m})$ foram tomadas, aleatoriamente, na área queimada. Todos os indivíduos lenhosos arbóreos vivos, com Db (diâmetro tomado a 0,30 m do solo) igual ou superior a $3 \mathrm{~cm}$, foram identificados botanicamente, e seus diâmetros e alturas foram registrados. Valores de densidade, dominância, freqüência e índice de valor de cobertura foram obtidos para os dois levantamentos. A avaliação da similaridade florística entre os dois inventários foi realizada para dados qualitativos (presença e ausência de espécies), a partir do índice de Sфrensen. Os resultados mostraram que houve pouca mudança na composição florística da comunidade durante o período estudado. Entre os dois inventários houve um aumento de aproximadamente $125 \%$ na densidade por hectare. A similaridade entre os dois levantamentos foi alta (índice de S申rensen igual a 0,68). A densidade florística, obtida a partir do índice de Shannon, foi baixa (cerca de 2,5) nas duas épocas monitoradas, quando comparada com pesquisas a longo prazo no cerrado sensu stricto da FAL (cerca de 3,5). Contudo, este valor é comum em áreas de cerrado que sofreram distúrbios. No segundo inventário, realizado três anos após o fogo, surgiram 13 espécies novas na área, o que comprova a sua recuperação e recolonização.
\end{abstract}

Palavras-chave: Incêndio florestal, composição florística, cerrado e fitossociologia.

\section{EFFECT OF FIRE ONTHE STRUCTURE AND FLORISTIC COMPOSITION OF A CERRADO SENSU STRICTO AREA IN FAZENDA ÁGUA LIMPA-DF}

\begin{abstract}
This research aimed to evaluate the effect of fire on the structure and floristic composition of a 10 ha cerrado sensu stricto area located at the Ecological and Experimental Reserve of the University of Brasilia, Fazenda Água Limpa-FAL. Two assessments were conducted in the area: the first, after the area had suffered a criminal fire in 1999, and the second in 2002. A sample of four $20 \times 50 \mathrm{~m}$ permanent plots was assessed. All individuals with stems $5 \mathrm{~cm}$ diameter at $0,30 \mathrm{~m}(\mathrm{Db})$ from the ground level were registered, and had their $\mathrm{Db}$ and total height measured. Density, basal area, frequency and value of covering index were calculated for the two assessments. The floristic similarity between the two surveys was evaluated for qualitative data (presence and absence of species) based on the Sфrensen Index. Few changes were observed in the floristic composition of the community during the studied period. There was an increase of $125 \%$ in density by hectare from 1999 to 2002. A high similarity (Sprensen Index equal to 0.68)was found between the two assessments while diversity (Shannon Index) was small (about 2.5). This value is smaller than those found in natural areas of the Cerrado sensu stricto at Fazenda Água Limpa, although it is common in areas with disturbances. Three years after fire 13 new species were verified in the area.
\end{abstract}

Key words: $\quad$ Forest fire, floristic composition, cerrado, phytosociology.

1 Recebido para publicação em 2.5.2003 e aceito para publicação em 17.2.2004.

Trabalho financiado pelo CNPq.

2 Departamento de Engenharia Florestal da Universidade de Brasília - UnB, Caixa Postal 04357, 70910-900 Brasília-DF, <fiedler@unb.br>. ${ }^{3}$ Pesquisador EMBRAPA-CENARGEN, Brasília-DF, <medeiros@ cenargen.embrapa.br>. ${ }^{4}$ Eng. Florestal, NOVACAP, Brasília-DF, <venturoli@ rudah.com.br>. 


\section{INTRODUÇÃO}

O cerrado é o segundo maior bioma brasileiro, e recentemente foi incluído na lista dos "hotspots" (Silva $\&$ Bates, 2002). Por um lado, a inclusão do bioma cerrado nesta lista tem um ponto positivo, pois significa o reconhecimento, em nível mundial, de sua rica biodiversidade. Por outro lado, sua inclusão também mostra que ele está sendo considerado um ambiente bastante ameaçado e que a sobrevivência de suas espécies depende de sua conservação e preservação.

A grande heterogeneidade de paisagens, característica do cerrado, abriga uma importante diversidade florística (Ratter et al. 2000), com um significativo número de espécies de plantas endêmicas (Giulietti et al. 2000).

De modo geral, o bioma cerrado encontra-se bastante ameaçado. Espécies nativas importantes, comercial e ecologicamente, estão desaparecendo em função da ocupação desordenada, da expansão urbana e agropecuária, da exploração irracional e do uso indiscriminado do fogo.

Os distúrbios na vegetação do cerrado têm ocorrido em vastas áreas. Hoje, menos de 3\% de sua superfície está protegida em Unidades de Conservação e mais de $60 \%$ da região já foi profundamente modificada pela ocupação antrópica, ou seja, lavouras diversas, pastagens e ocupação urbana (Brasil et al., 1999; Cavalcanti, 2000).

As queimadas naturais provocadas por raios nesse ecossistema, apesar de pouco estudadas, são relativamente freqüentes, ocorrendo principalmente na transição seca-chuva. Apesar da alta freqüência destas queimadas, elas geralmente não atingem grandes áreas, uma vez que a chuva posterior impede o desenvolvimento de incêndios de maior magnitude (Ramos-Neto, 2000; Fiedler \& Medeiros, 2002). Há também evidências de que estas queimadas podem tornar as comunidades da flora mais heterogêneas (Ramos-Neto, 2000).

Entre as características que reforçam a idéia de estratégias adaptativas da vegetação do cerrado ao fogo, estão a forte suberização do tronco e dos galhos, o que permite certo grau de isolamento térmico dos tecidos internos, mesmo em temperaturas elevadas (Guedes, 1993; Coutinho, 1990), e também a capacidade que os frutos têm de proteger as sementes (Landim \& Hay, 1995; Cirne, 2002). Já foram verificados efeitos positivos do fogo sobre a dispersão de sementes (Coutinho, 1977) e em relação à indução de floração do estrato herbáceo
(Coutinho, 1976; Oliveira et al., 1996). Entre as espécies lenhosas, é comum a rebrota através da copa, dos rizomas, do caule, da raiz e das estruturas subterrâneas (Souza \& Soares, 1983; Coutinho, 1990; Medeiros, 2002).

O fogo pode ter também efeitos negativos para a vegetação do cerrado, dependendo da sua freqüência e intensidade. Entre os efeitos adversos de queimadas freqüentes no cerrado, já foi observado o baixo recrutamento de espécies lenhosas, cujas conseqüências foram a redução na densidade arbórea, o aumento do entouceiramento e a diminuição da diversidade de espécies (Sambuichi, 1991). Em suas pesquisas Medeiros (2002), Silva et al. (1996), Silva (1999) e Sato (1996) verificaram elevadas taxas de mortalidade em campo-sujo e no cerrado sensu stricto após queimadas prescritas.

Miranda et al. (1996) observaram, durante duas queimadas em uma área de campo-sujo, que $97 \%$ do material consumido era de herbáceas e que as máximas temperaturas $\left(604\right.$ e $\left.752{ }^{\circ} \mathrm{C}\right)$ foram registradas a $60 \mathrm{~cm}$ de altura do solo, com duração de 120 segundos.

O fogo colocado, visando a renovação de pastagens e a limpeza de áreas, é em geral realizado sem a mínima preocupação com a segurança, tanto na área a ser queimada quanto no entorno. Neste caso, além da necessidade de obediência aos aspectos legais para realização da queima controlada (PORTARIA № 94/98-N, de 9 de julho de 1998, que regulamenta procedimentos referentes a queimadas), devem ser tomados alguns cuidados, como a construção de aceiros, a execução de queima contra o vento e a presença de uma equipe treinada em combate ao fogo em caso de perda do controle da situação.

Conhecimentos sobre florística, fitossociologia e ecologia do cerrado são extremamente necessários para elaboração de propostas para recuperação de áreas que sofreram distúrbios devido à ocorrência de incêndios florestais. Portanto, o objetivo desta pesquisa foi avaliar o efeito de um incêndio florestal na estrutura e na composição florística da vegetação arbórea de um cerrado sensu stricto, na fazenda Água Limpa-DF.

\section{MATERIAL E MÉTODOS}

\section{1. Área de Estudo}

Esta pesquisa foi realizada em uma área de 10 ha de cerrado sensu stricto, localizada na Reserva Ecológica e Experimental da Universidade de Brasília, fazenda Água 
Limpa - FAL, situada a $30 \mathrm{~km}$ do Plano Piloto de BrasíliaDF, a uma altitude de $1.100 \mathrm{~m}$, nas coordenadas $15^{\circ} 56^{\prime}-$ $15^{\circ} 59^{\prime} \mathrm{S}$ e $47^{\circ} 55^{\prime}-47^{\circ} 58^{\prime}$ WGr. A fazenda Água Limpa tem uma área total de 4.390,0 ha, e o solo predominante na área de estudo é o Latossolo Vermelho-Amarelo, pobre em nutrientes e com um alto teor de alumínio. O clima da região é do tipo Aw, segundo a classificação de Köppen, com temperatura máxima de $28,5^{\circ} \mathrm{C}$ e mínima de $12{ }^{\circ} \mathrm{C}$. A umidade relativa entre maio e setembro fica abaixo de $70 \%$ e a umidade mínima ocorre em agosto, com uma média de $47 \%$, podendo cair para $15 \%$ nos períodos mais secos. A precipitação média anual é de $1.600 \mathrm{~mm}$, com uma pronunciada estação seca de junho a setembro.

O cerrado sensu stricto é a vegetação predominante e ocupa uma área de 148 ha. A flora local é rica e inclui espécies raras e endêmicas (Felfili et al., 1993).

O fogo no cerrado da fazenda Água Limpa ocorre, em geral, a cada cinco anos, e registros indicam que a temperatura média do ar a $60 \mathrm{~cm}$ de altura é igual a $600^{\circ} \mathrm{C}$. A velocidade da frente de fogo é variável, mas, em média, encontra-se na faixa de $0,4 \mathrm{~m} / \mathrm{s}$. Tais valores são similares aos encontrados em queimadas de cerrado e de savanas (Miranda et al., 1993; Frost \& Robertson 1984).

\subsection{Coleta dos Dados}

Para esta pesquisa foi selecionada uma área de 10 ha de cerrado sensu stricto, que foi totalmente queimada no dia 7 de setembro de 1999, por um incêndio florestal criminoso.

Cerca de 30 dias após o fogo, foram selecionadas, aleatoriamente, quatro parcelas de 0,1 ha $(20 \times 50 \mathrm{~m})$ na área queimada. Todos os indivíduos lenhosos arbóreos vivos e mortos em pé, com Db (diâmetro tomado a $0,30 \mathrm{~m}$ do solo) igual ou superior a $3 \mathrm{~cm}$, foram identificados botanicamente e etiquetados, e seus diâmetros e alturas foram registrados. A identificação botânica dos indivíduos foi possível, considerando que a grande maioria das espécies é adaptada ao fogo e cresce rapidamente após a ocorrência de um incêndio, a partir de meristemas bem protegidos na árvore (Vieira et al., 1996). Além disto, indivíduos já estabelecidos não sofrem com a ação do fogo. Segundo Ramos (1990), a mortalidade de plantas estabelecidas devido ao fogo é um fenômeno raro ou somente manifestado a longo prazo, uma vez que as queimas sucessivas podem lesar tecidos vegetais e facilitar o ataque de pragas e doenças. Esta característica é também observada em plantas já estabelecidas das savanas africanas.

Em novembro de 2002 ( 3 anos e 2 meses após o incêndio), foi realizado um segundo levantamento nas mesmas parcelas, sendo medidos todos indivíduos vivos e mortos, em pé, registrados no inventário de 1999, além de medir, identificar e etiquetar os novos indivíduos encontrados, com os mesmos parâmetros utilizados no primeiro inventário.

Neste estudo, considerou-se árvore morta todo indivíduo lenhoso arbóreo-arbustivo com $\mathrm{Db} \geq 3 \mathrm{~cm}$ que apresentava toda a parte aérea morta, em cada época monitorada. Indivíduos classificados como mortos no primeiro monitoramento, mas que se regeneraram posteriormente, a partir de raízes, foram considerados como recrutas no segundo levantamento, realizado em 2002.

\subsection{Análise dos Dados}

Para verificar as modificações na composição florística e na estrutura da vegetação do cerrado em decorrência da ação do fogo, foi realizada uma comparação da composição florística e dos índices que expressam a estrutura horizontal da vegetação, ou seja, densidade, dominância, freqüência e índice de valor de cobertura. Estes foram obtidos tanto para o levantamento realizado em 1999 (imediatamente após o fogo) quanto para o levantamento realizado em 2002.

A avaliação da similaridade florística entre os dois inventários foi realizada para dados qualitativos (presença e ausência de espécies), a partir do índice de Sørensen (Kent \& Coker, 1992), que é dado por:

$$
S_{s}=\frac{2 a}{2 a+b+c} \quad 0 \leq S_{s} \leq 1
$$

em que $a=$ número de espécies comuns em ambos os levantamentos; $b=$ número de espécies registradas no levantamento de 1999; e $c=$ número de espécies registradas no levantamento de 2002.

Esse índice varia de 0 (completa dissimilaridade) a 1 ou $100 \%$ (total similaridade).

A diversidade florística, imediatamente após o fogo e três anos e dois meses após a sua ocorrência, foi obtida a partir do índice de Shannon (H') (Kent \& Coker, 1992), que é dado por: 


$$
H^{\prime}=-\sum_{i=1}^{s} p_{i} \ln p_{i}
$$

em que $s=$ número de espécies; $p_{i}=$ proporção de indivíduos ou abundância da i-ésima espécie expressa como uma proporção da cobertura total; e $l n=$ logaritmo na base $n(n=2,10$ ou $e)$.

O índice de Shannon expressa a riqueza florística de uma amostra ou comunidade e assume que todas as espécies estão representadas na amostra. Seu valor geralmente encontra-se entre 1,5 e 3,5, embora em casos excepcionais possa exceder 4,5 .

\section{RESULTADOS E DISCUSSÃO}

\subsection{Composição Florística e Estrutura da Vegetação}

Nos Quadros 1 e 2 estão as listas de espécies lenhosas arbóreo-arbustivas encontradas no cerrado sensu stricto estudado nos dois levantamentos realizados.

No total, foram registradas na área 46 espécies, pertencentes a 23 famílias. As famílias mais ricas em espécies foram Leguminosae e Vochysiaceae. Do total de famílias encontradas, $48 \%$ foram representadas por apenas uma espécie.

Em 1999, imediatamente após a ocorrência do fogo, foram registrados, em 0,4 ha, 294 indivíduos com $\mathrm{Db} \geq$ $3 \mathrm{~cm}$, distribuídos em 34 espécies e 27 gêneros, pertencentes a 15 famílias. A família Leguminosae teve o maior número de espécies (7), seguida da família Vochysiaceae (5). A família Leguminosae também se destacou quanto ao número de indivíduos (50\%).

Em 2002, três anos após a ocorrência do fogo, foram registrados 666 indivíduos na mesma área ( $0,4 \mathrm{ha})$, distribuídos em 40 espécies e 33 gêneros, pertencentes a 20 famílias. Contudo, mesmo tendo sido observado um aumento significativo no número de espécies (18\%), a família Leguminosae continuou sendo a mais rica em espécies (7), seguida das famílias Vochysiaceae (4) e Malpighiaceae (4). Tais famílias também se destacaram quanto ao número de indivíduos, tendo Leguminosae se sobressaído principalmente devido à espécie arbustiva Mimosa claussenii, que dominava o local. Segundo Rezende (2002), essa espécie ocorre em abundância logo após distúrbios por fogo em áreas de cerrado. Contudo, ela apresenta ciclo de vida curto e pode ser classificada como uma espécie oportunista, cuja grande ocorrência após o primeiro levantamento pode ser em função de um outro incêndio na área, em 1996.

Houve mudança na composição florística da comunidade durante o período estudado, com o surgimento de 13 novas espécies. A grande maioria das espécies registradas no primeiro inventário e que não foram encontradas no segundo são aquelas com baixa densidade e, portanto, muito mais suscetíveis de serem eliminadas da área, a não ser que haja algum recrutamento. Com a presença do fogo, a probabilidade de muitas espécies, principalmente as mais jovens, não resistirem e desaparecerem é muito grande, e provavelmente os indivíduos relacionados a estas espécies levariam maior tempo para se estabelecer na área novamente.

Na Figura 1, referente à curva espécie/área, nota-se um deslocamento ascendente da curva 1 (primeiro inventário) para a curva 2 (segundo inventário). A curva espécie/área 2 é ascendente, embora com tendência à estabilização. Este comportamento é resultante do recrutamento de espécies novas na área, conforme mencionado no parágrafo anterior.

O número de indivíduos por hectare passou de 737 para 1.665, o que mostra que a população na área dobrou. $\mathrm{O}$ aumento de densidade de indivíduos lenhosos após a ocorrência do fogo foi observado em outros estudos (Sambuichi, 1991; Moreira, 1992). Do total dos indivíduos, $70 \%$ estava na faixa de Db entre 3 e $7 \mathrm{~cm}$, e o maior diâmetro foi observado para Sclerolobium paniculatum, com $21,6 \mathrm{~cm}$.

Os indivíduos mortos encontrados estavam na faixa de diâmetro de 3 a $9 \mathrm{~cm}$ no primeiro inventário e de 3,5 a $14 \mathrm{~cm}$ no segundo, apresentando uma média de 3,9 e $7,3 \mathrm{~cm}$, respectivamente (Figura 2).

Observa-se (Figura 2) que no primeiro inventário (1999) 76\% dos indivíduos mortos tinham entre 3 e $4 \mathrm{~cm}$ de diâmetro. Já no segundo (2002), a maior mortalidade foi observada para indivíduos entre 4,1 e $5 \mathrm{~cm}$ de diâmetro (26\%).

Dentre as espécies que se destacaram por apresentar altas taxas de mortalidade nos dois períodos monitorados e que apresentavam diâmetros variando de 3 a $5 \mathrm{~cm}$, destacam-se: Ouratea hexasperma, Davilla elliptica, Styrax ferrugineus, Roupala montana, Qualea sp., Erythroxylum deciduum e Erythroxylum tortuosum. 
Quadro 1 - Variáveis fitossociológicas encontradas para o cerrado sensu stricto inventariado em 1999, na fazenda Água Limpa-FAL, DF, após a ocorrência do incêndio

Table 1 - Phytosociology variables found for Cerrado sensu stricto assessed in 1999 at Fazenda Água Limpa after the forest fire

\begin{tabular}{|c|c|c|c|c|c|c|c|c|c|}
\hline Família & Espécie & № & DA & DR & FA & FR & DoA & DoR & VI \\
\hline Leguminosae & Mimosa claussenii Benth. & 117 & 292,5 & 39,66 & 100 & 5,55 & 0,31 & 32,01 & 77,22 \\
\hline Vochysiaceae & Qualea parviflora Mart. & 23 & 57,5 & 7,80 & 75 & 4,16 & 0,07 & 6,90 & 18,86 \\
\hline Malpighiaceae & Byrsonima crassa Nied. & 21 & 52,5 & 7,12 & 75 & 4,16 & 0,07 & 7,24 & 18,53 \\
\hline Vochysiaceae & Qualea grandiflora Mart. & 14 & 35,0 & 4,75 & 75 & 4,16 & 0,06 & 5,75 & 14,66 \\
\hline Leguminosae & Machaerium acutifolium Vogel & 15 & 37,5 & 5,08 & 75 & 4,16 & 0,04 & 4,25 & 13,50 \\
\hline Rubiaceae & Tocoyena formosa K. Schum. & 2 & 5,0 & 0,68 & 25 & 1,38 & 0,07 & 7,76 & 9,83 \\
\hline Vochysiaceae & Vochysia thyrsoidea Pohl & 9 & 22,5 & 3,05 & 75 & 4,16 & 0,02 & 1,96 & 9,17 \\
\hline Leguminosae & Sclerolobium paniculatum Vogel & 3 & 7,5 & 1,02 & 50 & 2,77 & 0,04 & 4,99 & 8,78 \\
\hline Guttiferae & Kielmeyera coriacea Mart. & 8 & 20,0 & 2,71 & 75 & 4,16 & 0,02 & 1,62 & 8,50 \\
\hline Leguminosae & Dalbergia miscolobium Benth. & 7 & 17,5 & 2,37 & 50 & 2,77 & 0,03 & 3,05 & 8,20 \\
\hline Erythroxylaceae & Erythroxylum suberosum A.St.-Hil. & 5 & 12,5 & 1,69 & 75 & 4,16 & 0,02 & 2,32 & 8,19 \\
\hline Apocynaceae & Aspidosperma tomentosum Mart. & 7 & 17,5 & 2,37 & 75 & 4,16 & 0,02 & 1,62 & 8,16 \\
\hline Proteaceae & Roupala montana Aubl. & 7 & 17,5 & 2,37 & 75 & 4,16 & 0,02 & 1,52 & 8,06 \\
\hline Malpighiaceae & Byrsonima coccolobifolia H.B.K. & 7 & 17,5 & 2,37 & 50 & 2,77 & 0,02 & 2,13 & 7,28 \\
\hline Bombacaceae & Eriotheca pubescens (Mart. \& Zucc.) Schott \& Endl. & 3 & 7,5 & 1,02 & 50 & 2,77 & 0,03 & 2,78 & 6,58 \\
\hline Myrtaceae & Guapira noxia (Netto) Lundell & 3 & 7,5 & 1,02 & 75 & 4,16 & 0,01 & 1,37 & 6,55 \\
\hline Compositae & Eremanthus glomerulatus Less. & 5 & 12,5 & 1,69 & 50 & 2,77 & 0,01 & 1,27 & 5,75 \\
\hline Guttiferae & Kielmeyera speciosa A.St.-Hil. & 3 & 7,5 & 1,02 & 75 & 4,16 & 0,01 & 0,56 & 5,74 \\
\hline Leguminosae & Stryphnodendron adstringens (Mart.) Coville & 4 & 10,0 & 1,36 & 50 & 2,77 & 0,01 & 1,54 & 5,67 \\
\hline Leguminosae & Bauhinia rufa Steud. & 3 & 7,5 & 1,02 & 50 & 2,78 & 0,01 & 1,43 & 5,23 \\
\hline Lythraceae & Lafoensia pacari A. St.-Hil. & 4 & 10,0 & 1,36 & 50 & 2,77 & 0,01 & 0,96 & 5,09 \\
\hline Bignoniaceae & Tabebuia caraiba (Mart.) Bureau & 4 & 10,0 & 1,36 & 50 & 2,77 & 0,01 & 0,86 & 5,00 \\
\hline Solanaceae & Solanum lycocarpum A.St.-Hil. & 2 & 5,0 & 0,68 & 50 & 2,77 & 0,01 & 0,70 & 4,16 \\
\hline Leguminosae & Dimorphandra mollis Benth. & 2 & 5,0 & 0,68 & 50 & 2,77 & 0,00 & 0,43 & 3,88 \\
\hline Myrtaceae & Psidium myrsinoides Berg & 2 & 5,0 & 0,68 & 50 & 2,77 & 0,00 & 0,36 & 3,82 \\
\hline Vochysiaceae & Vochysia elliptica Mart. & 4 & 10,0 & 1,36 & 25 & 1,38 & 0,01 & 0,84 & 3,58 \\
\hline Hippocrateaceae & Salacia elliptica G. Don & 2 & 5,0 & 0,68 & 25 & 1,38 & 0,01 & 1,41 & 3,48 \\
\hline Celastraceae & Austroplenckia populnea (Reissek) Lundell & 2 & 5,0 & 0,68 & 25 & 1,38 & 0,00 & 0,38 & 2,44 \\
\hline Bignoniaceae & Tabebuia ochracea (Cham.) Standl. & 1 & 2,5 & 0,34 & 25 & 1,38 & 0,01 & 0,53 & 2,26 \\
\hline Connaraceae & Connarus suberosus Planch. & 1 & 2,5 & 0,34 & 25 & 1,38 & 0,00 & 0,29 & 2,02 \\
\hline Dilleniaceae & Davilla elliptica A.St.-Hill & 1 & 2,5 & 0,34 & 25 & 1,38 & 0,00 & 0,19 & 1,92 \\
\hline Hippocrateaceae & Salacia crassifolia G. Don & 1 & 2,5 & 0,34 & 25 & 1,38 & 0,00 & 0,18 & 1,91 \\
\hline Ebenaceae & Diospyros burchellii DC. & 1 & 2,5 & 0,34 & 25 & 1,38 & 0,00 & 0,18 & 1,91 \\
\hline Vochysiaceae & Qualea multiflora Mart. & 1 & 2,5 & 0,34 & 25 & 1,38 & 0,00 & 0,18 & 1,91 \\
\hline \multicolumn{2}{|r|}{ Total Global } & 294 & 737 & 100 & 1.800 & 100 & 0,957 & 100 & 300 \\
\hline
\end{tabular}

№ = número absoluto de indivíduos registrados, $\mathrm{DA}=$ densidade absoluta por hectare, $\mathrm{DR}=$ densidade relativa; $\mathrm{FA}=$ freqüência absoluta, $\mathrm{FR}=$ freqüência relativa; DoA = dominância absoluta por hectare, $\mathrm{DoR}=$ dominância relativa por hectare e VI = valor de importância. 
Quadro 2 - Variáveis fitossociológicas encontradas para o cerrado sensu stricto inventariado em 2002 na fazenda Água Limpa, FAL, DF, três anos após o incêndio

Table 2 - Phytosociol ogy variables found for Cerrado sensu stricto assessed in 2002 at Fazenda Água Limpa after theforest fire

\begin{tabular}{|c|c|c|c|c|c|c|c|c|c|}
\hline Família & Espécie & $\mathrm{N}^{\mathrm{o}}$ & DA & DR & FA & FR & DoA & DoR & VI \\
\hline Leguminosae & Mimosa clausennii & 219 & 547,5 & 32,88 & 100 & 4,40 & 0,69 & 18,10 & 55,38 \\
\hline Vochysiaceae & Qualea parviflora & 96 & 240,0 & 14,41 & 100 & 4,40 & 0,47 & 12,30 & 31,11 \\
\hline Malpighiaceae & Byrsonima crassa & 86 & 215,0 & 12,91 & 100 & 4,40 & 0,45 & 11,97 & 29,28 \\
\hline Leguminosae & Sclerolobium paniculatum & 32 & 80,0 & 4,80 & 75 & 3,30 & 0,37 & 9,84 & 17,95 \\
\hline Leguminosae & Stryphnodendron adstringens & 26 & 65,0 & 3,90 & 100 & 4,40 & 0,19 & 4,98 & 13,28 \\
\hline Malpighiaceae & Byrsonima coccolobifolia & 31 & 77,5 & 4,65 & 100 & 4,40 & 0,13 & 3,54 & 12,59 \\
\hline Vochysiaceae & Vochysia thyrsoidea & 5 & 12,5 & 0,75 & 50 & 2,20 & 0,33 & 8,76 & 11,71 \\
\hline Vochysiaceae & Vochysia elliptica & 8 & 20,0 & 1,20 & 50 & 2,20 & 0,31 & 8,20 & 11,60 \\
\hline Bombacaceae & Eriotheca pubescens & 16 & 40,0 & 2,40 & 75 & 3,30 & 0,20 & 5,31 & 11,00 \\
\hline Guttiferae & Kielmeyera coriacea & 20 & 50,0 & 3,00 & 100 & 4,40 & 0,06 & 1,62 & 9,02 \\
\hline Guttiferae & Kielmeyera speciosa & 14 & 35,0 & 2,10 & 100 & 4,40 & 0,06 & 1,61 & 8,11 \\
\hline Leguminosae & Machaerium acutifolium & 15 & 37,5 & 2,25 & 75 & 3,30 & 0,08 & 2,23 & 7,78 \\
\hline Rubiaceae & Tocoyena formosa & 10 & 25,0 & 1,50 & 100 & 4,40 & 0,03 & 0,91 & 6,81 \\
\hline Apocynaceae & Aspidosperma tomentosum & 7 & 17,5 & 1,05 & 100 & 4,40 & 0,01 & 0,51 & 5,95 \\
\hline Myrsinaceae & Rapanea guianensis Aubl. & 15 & 37,5 & 2,25 & 50 & 2,20 & 0,05 & 1,44 & 5,89 \\
\hline Lythraceae & Lafoensia pacari & 8 & 20,0 & 1,20 & 75 & 3,30 & 0,03 & 0,85 & 5,34 \\
\hline Erythroxylaceae & Erythroxylum suberosum & 7 & 17,5 & 1,05 & 75 & 3,30 & 0,02 & 0,63 & 4,98 \\
\hline Dilleniaceae & Davilla elliptica & 5 & 12,5 & 0,75 & 75 & 3,30 & 0,03 & 0,78 & 4,83 \\
\hline Celastraceae & Austroplenckia populnea & 5 & 12,5 & 0,75 & 75 & 3,30 & 0,01 & 0,43 & 4,48 \\
\hline Nyctaginaceae & Guapira noxia & 4 & 10,0 & 0,60 & 75 & 3,30 & 0,01 & 0,51 & 4,40 \\
\hline Myrtaceae & Psidium myrsinoides & 5 & 12,5 & 0,75 & 75 & 3,30 & 0,01 & 0,35 & 4,40 \\
\hline Caryocaraceae & Caryocar brasiliense Cambess. & 6 & 15,0 & 0,90 & 50 & 2,20 & 0,04 & 1,15 & 4,25 \\
\hline Hippocrateaceae & Salacia crassifolia & 2 & 5,0 & 0,30 & 50 & 2,20 & 0,00 & 0,16 & 2,65 \\
\hline Sapotaceae & Pouteria ramiflora Radlk. & 2 & 5,0 & 0,30 & 25 & 1,10 & 0,04 & 1,25 & 2,65 \\
\hline Malpighiaceae & Byrsonima verbascifolia Rich. ex A. Juss. & 2 & 5,0 & 0,30 & 50 & 2,20 & 0,00 & 0,14 & 2,64 \\
\hline Apocynaceae & Aspidosperma macrocarpon Mart. & 3 & 7,5 & 0,45 & 25 & 1,10 & 0,01 & 0,32 & 1,87 \\
\hline Bignoniaceae & Tabebuia caraiba & 1 & 2,5 & 0,15 & 25 & 1,10 & 0,02 & 0,51 & 1,76 \\
\hline Compositae & Eremanthus glomerulatus & 2 & 5,0 & 0,30 & 25 & 1,10 & 0,00 & 0,25 & 1,65 \\
\hline Apocynaceae & Hancornia speciosa Gomez & 2 & 5,0 & 0,30 & 25 & 1,10 & 0,00 & 0,22 & 1,62 \\
\hline Malpighiaceae & Heteropterys byrsonimaefolia A. Juss. & 2 & 5,0 & 0,30 & 25 & 1,10 & 0,00 & 0,16 & 1,56 \\
\hline Leguminosae & Hymenaea stignocarpa Mart. & 1 & 2,5 & 0,15 & 25 & 1,10 & 0,00 & 0,24 & 1,49 \\
\hline Ochnaceae & Ouratea hexasperma Baill. & 1 & 2,5 & 0,15 & 25 & 1,10 & 0,00 & 0,10 & 1,35 \\
\hline Proteaceae & Roupala montana & 1 & 2,5 & 0,15 & 25 & 1,10 & 0,00 & 0,10 & 1,35 \\
\hline Myrtaceae & Blepharocalyx salicifolius (Kunth) O. Berg & 1 & 2,5 & 0,15 & 25 & 1,10 & 0,00 & 0,10 & 1,35 \\
\hline Leguminosae & Plathymenia reticulata Benth. & 1 & 2,5 & 0,15 & 25 & 1,10 & 0,00 & 0,10 & 1,35 \\
\hline Erythroxylaceae & Erythroxylum tortuosum Mart. & 1 & 2,5 & 0,15 & 25 & 1,10 & 0,00 & 0,08 & 1,33 \\
\hline Bignoniaceae & Tabebuia ochracea & 1 & 2,5 & 0,15 & 25 & 1,10 & 0,00 & 0,07 & 1,32 \\
\hline Erythroxylaceae & Erythroxylum deciduum A.St.-Hil. & 1 & 2,5 & 0,15 & 25 & 1,10 & 0,00 & 0,07 & 1,32 \\
\hline Vochysiaceae & Qualea grandiflora & 1 & 2,5 & 0,15 & 25 & 1,10 & 0,00 & 0,06 & 1,31 \\
\hline Leguminosae & Dimorphandra mollis & 1 & 2,5 & 0,15 & 25 & 1,10 & 0,00 & 0,05 & 1,30 \\
\hline \multicolumn{2}{|r|}{ Total Global } & 666 & 1.665 & 100 & 2.275 & 100 & 3.818 & 100 & 300 \\
\hline
\end{tabular}

$\mathrm{N}^{\mathrm{o}}=$ número absoluto de indivíduos registrados, $\mathrm{DA}=$ densidade absoluta por hectare, $\mathrm{DR}=$ densidade relativa, $\mathrm{FA}=$ frequiência absoluta, $\mathrm{FR}=$ frequiência relativa, $\mathrm{DoA}=$ dominância absoluta por hectare, $\mathrm{DoR}=$ dominância relativa por hectare e $\mathrm{VI}=$ índice de valor de importância.

R. Árvore, Viçosa-MG, v.28, n.1, p.129-138, 2004 


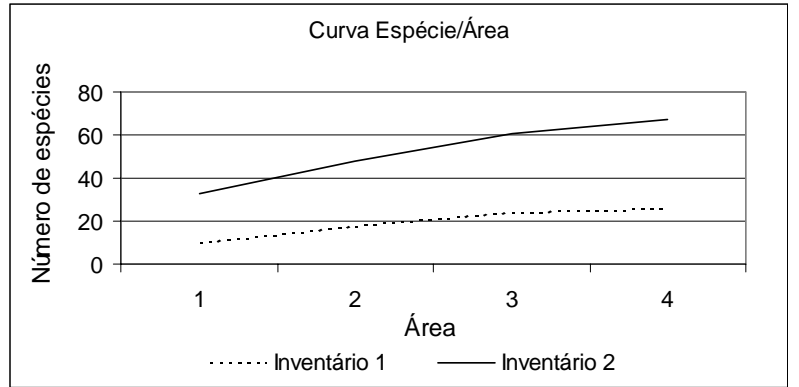

Figura 1 - Curva espécie/área nos dois inventários realizados em cerrado sensu stricto na fazenda Água Limpa-FAL, DF.

Figure 1 - Species/ area curvein thetwo assessments carried out in a Cerrado sensu stricto at Fazenda Água Limpa, DF.

Medeiros (2002), em inventário realizado na Reserva Ecológica do IBGE em Brasília, mediu plantas a partir de 2,0 cm de diâmetro, ou seja, incluiu na sua amostragem indivíduos de menor porte, que são mais propensos aos danos causados pelo fogo. Ele estimou taxas de mortalidade entre 8,2 , e $22,5 \%$ após queimadas anuais sucessivas. Os dados encontrados pelo autor mostraram que o diâmetro dos indivíduos é um fator determinante para a sobrevivência e que mesmo plantas não muito altas podem sobreviver caso o diâmetro do caule (maior) determine maior resistência. De acordo com os dados analisados, houve maior porcentagem de mortalidade nas classes de diâmetro menores (entre 2 e
$3 \mathrm{~cm}$ ): os indivíduos com diâmetros superiores a $5 \mathrm{~cm}$ apresentaram chances maiores de sobrevivência. Inventários que consideram indivíduos de menor porte podem representar melhor a amostra, considerando que para a distribuição de indivíduos de plantas lenhosas no cerrado é maior o número nas classes de diâmetro inferiores a $5 \mathrm{~cm}$ (Ottmar et al., 2001). Em pesquisas realizadas em áreas de cerrado, Miranda et al. $(1993,1996)$ concluíram que o calor desprendido pelo fogo durante as queimadas afeta, principalmente, os indivíduos situados a altura na faixa de $60 \mathrm{~cm}$, onde ocorre a zona crítica de temperatura. Assim, indivíduos com altura inferior a 2,0 m têm maior exposição da copa às maiores temperaturas.

Medeiros (2002) constatou elevadas taxas de mortalidade para algumas espécies após queimadas freqüentes, o que caracteriza estas espécies como sendo sensíveis à ocorrência freqüente de fogo. Dentre estas espécies destacam-se Styrax ferrugineus, Erythroxylum deciduum, Erythroxylum tortuosum, Roupala montana, Acosmium dasycarpum e Rourea induta. Outras espécies como Byrsonima verbascifolia, Davilla elliptica e Ouratea hexasperma apresentaram maior resistência. Com exceção de Byrsonima verbascifolia, Sato (1996) também encontrou dados similares para espécies mais resistentes e Moreira (1992) observou dados similares para Ouratea hexasperma. Felfilli et al. (2000) também observaram, em cerrado sensu stricto, elevada mortalidade para Styrax ferrugineus, Erythroxylum deciduum,

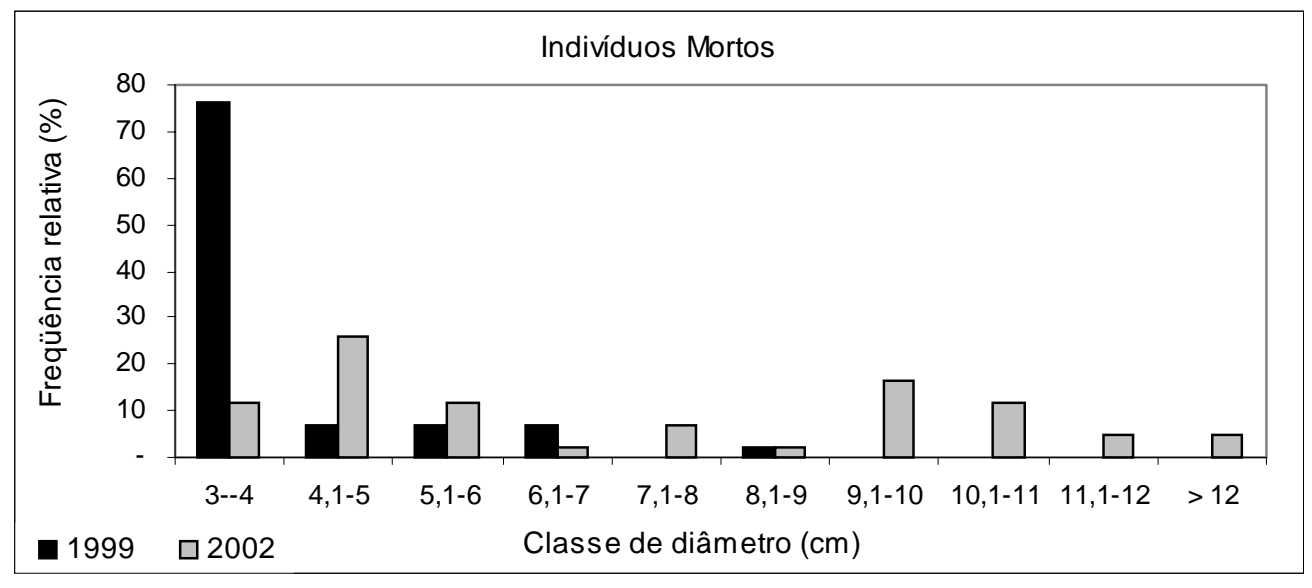

Figura 2 - Freqüência relativa por classe de diâmetro para os indivíduos que morreram após o fogo em uma área de cerrado sensu stricto na fazenda Água Limpa-FAL, DF, (no total de indivíduos mortos registrados em cada levantamento $=42$ ).

Figure 2 - Relative frequency per diameter class for the individuals thatdied after fire in an area of Cerrado sensu stricto at the Fazenda Água Limpa.

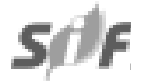

R. Árvore, Viçosa-MG, v.28, p.129-138, 2004 
Erythroxylum tortuosum, Roupala montana e Rourea induta. Em um estudo sobre dinâmica populacional de algumas espécies lenhosas do cerrado, Hoffmann (1999) verificou que Miconia albicans, Roupala montana, Periandra mediterranea e Myrsine guianensis devem sofrer um declínio nas suas populações no regime de fogo atual, ou seja, com intervalos de 1 a 3 anos.

A similaridade florística entre os dois levantamentos realizados (obtida a partir do índice de S申rensen) foi igual a 0,68 . Valores superiores a 0,5 indicam elevada similaridade entre as áreas.

O índice de diversidade (Shannon) encontrado no primeiro inventário foi de 2,55 e no segundo, de 2,47. Estes valores são menores que aqueles encontrados por Felfili \& Silva Jr. (1993) em áreas naturais de cerrado sensu stricto, ou seja, em Águas Emendadas $(3,62)$, Patrocínio $(3,53)$, APA Gama-Cabeça do Veado $(3,56)$, Parque Nacional de Brasília $(3,34)$, Silvânia $(3,31)$ e Paracatu $(3,11)$.

As espécies Dalbergia miscolobium, Bauhinia rufa, Solanum lycocarpum, Salacia elliptica, Connarus suberosus, Diospyros burchellii e Qualea multiflora não foram registradas no segundo inventário, no entanto, foram recrutadas novas espécies: Caryocar brasiliense, Pouteria ramiflora, Byrsonima verbascifolia, Aspidosperma macrocarpon, Hancornia speciosa, Heteropterys byrsonimaefolia, Hymenaea stigonocarpa, Ouratea hexasperma, Blepharocalyx salicifolius, Plathymenia reticulata, Erythroxylum tortuosum, Erythroxylum deciduum e Rapanea guianensis. Tais espécies são comuns na área estudada, e algumas delas ocorrem em altas densidades. A reincidência de queimadas na área provavelmente reduzirá o índice de diversidade. Sambuichi (1991) verificou que a elevada freqüência de fogo diminui a diversidade de elementos lenhosos no cerrado.

A riqueza florística encontrada na área de cerrado estudada foi menor do que a registrada por Felfili et al. (2000) no cerrado sensu stricto da fazenda Água Limpa, onde os autores avaliaram as modificações na composição florística desta área de cerrado durante o período de nove anos, a partir de um inventário contínuo realizado em 19 parcelas de 0,1 ha. Segundo os autores, em 1985 foram registrados na área 30 famílias, 50 gêneros e 61 espécies, e nove anos após (1994) havia 29 famílias, 47 gêneros e 57 espécies. Nos dois levantamentos as famílias Leguminosae, Vochysiaceae, Erythroxylaceae e Malpighiaceae foram as mais ricas em espécies.

R. Árvore, Viçosa-MG, v.28, n.1, p.129-138, 2004

\section{CONCLUSÕES}

a) Três anos após a ocorrência do fogo a densidade duplicou, indicando que a área está em fase de recuperação.

b) As espécies Dalbergia miscolobium, Bauhinia rufa, Solanum lycocarpum, Salacia elliptica, Connarus suberosus, Diospyros burchellii e Qualea multiflora não foram registradas no segundo inventário (três anos após o incêndio), o que pode indicar a baixa resistência destas ao fogo.

c) No segundo inventário surgiram as seguintes espécies: Caryocar brasiliense, Pouteria ramiflora, Byrsonima verbascifolia, Aspidosperma macrocarpon, Hancornia speciosa, Heteropterys byrsonimaefolia, Hymenaea stigonocarpa, Ouratea hexasperma, Blepharocalyx salicifolius, Plathymenia reticulata, Erythroxylum tortuosum, Erythroxylum deciduum e Rapanea guianensis, o que pode comprovar a recuperação e recolonização da área.

d) No cerrado sensu stricto, indivíduos lenhosos arbóreo-arbustivos com diâmetros inferiores a $4 \mathrm{~cm}$ foram mais suscetíveis ao fogo.

e) A riqueza florística do cerrado sensu stricto modificou-se após a ocorrência de fogo: algumas espécies, principalmente aquelas de baixa densidade, foram eliminadas da área e dependem de agentes dispersores para se reestabelecerem na área.

f) Estudos contínuos devem ser realizados para estudar a dinâmica do cerrado submetido a freqüentes incêndios.

\section{REFERÊNCIAS BIBLIOGRÁFICAS}

BRASIL. Ministério do Meio Ambiente, dos Recursos Hídricos e da Amazônia Legal. Ações prioritárias para a conservação da biodiversidade do Cerrado e Pantanal. Brasília: Ventura Comunicações e Cultura, 1999. 24 p.

CAVAlCANTI, R. Capricho da Natureza. UnB Revista, p. 20-23, 2000. Edição Especial.

CIRNE, P. Efeitos do fogo na regeneração de Kielmeyera coriacea (Spr.) Mart. (Guttiferae) em áreas de cerrado sensu stricto: mecanismos de sobrevivência e época de queima. 2002. 83 f. Tese (Doutorado em Ecologia) Universidade de Brasília, Brasília, 2002.

COUTINHO, L. M. Contribuição ao conhecimento do papel ecológico das queimadas na floração de espécies do cerrado. 1976. 123 f. Tese (Livre Docência) Universidade de São Paulo, São Paulo, 1976. 
COUTINHO, L. M. Aspectos ecológicos do fogo no cerrado. II - As queimadas e a dispersão de sementes em algumas espécies anemocóricas do estrato herbáceoarbustivo. Boletim de Botânica da Universidade de São Paulo, v. 5, p. 57-64, 1977.

COUTINHO, L. M. Fire in the ecology of the brazilian cerrado. In: GOLDAMMER, J. G. (Ed.) Fire in the Tropical Biota. Berlin: Springer-Verlag, 1990. p. 82-105.

FELFILI, J. M.; SILVA Jr., M. C. A comparative study of cerrado (sensu stricto) Vegetation in Central Brazil. Journal of Tropical Ecology, v. 9, p. 277-289, 1993.

FELFILLI, J. M. et al. Changes in the floristic composition of cerrado sensu stricto in Brazil over a nine-year period. Journal of Tropical Ecology, v. 16, p. 579-590, 2000.

FIEDLER, N. C.; MEDEIROS, M. B. Plano de prevenção e combate aos incêndios florestais no Parque Nacional da Serra da Canastra. In: Plano de Manejo do Parque Nacional da Serra da Canastra. IBAMA. Brasília. 67 p.

FROST, P. G.; ROBERTSON, F. The ecological effects of fire in savannas. In: WALKER, B. H. (Ed.). Determinants of Tropical Savannas. IRL Press, Oxford, 1987. p. 93-140.

GIULIETTI, A. M. et al. Caracterização e endemismos nos campos rupestres da cadeia do espinhaço. In: TÓPICOS Atuais em Botânica. Sociedade Botânica do Brasil/Embrapa Recursos Genéticos e Biotecnologia. Brasília, 2000. p. 311 318.

GUEDES, D. M. Resistência das árvores do cerrado ao fogo: papel da casca como isolante térmico. 1993. $113 \mathrm{f}$. Dissertação (Mestrado em Ecologia) - Universidade de Brasília, Brasília, 1993.

HOFFMANN, W. A. Fire and population dynamics of woody plants in a neotropical savanna: matrix model projections. Ecology, v. 80, n. 4, p. 1354-1369, 1999.

KENT, M.; COKER, P. Vegetation Description and Analysis: a practical approach. New York: John Wiley \& Sons, 1992. $363 \mathrm{p}$.

LANDIM, M. F.; HAY, J. D. Impacto do fogo sobre alguns aspectos da biologia reprodutiva de Kielmeyera coriacea Mart. Revista Brasileira de Biologia, v. 56, n. 1, 127-134, 1995.

MEDEIROS, M. B. Efeitos do fogo nos padrões de rebrotamento em plantas lenhosas, em campo sujo. 2002. $121 \mathrm{f}$. Tese (Doutorado em Ecologia) - Universidade de Brasília, Brasília, 2002.
MIRANDA, A. C. et al. Soil and air temperatures during prescribed Cerrado fires in Central Brazil. Journal of Tropical Ecology, v. 9, p. 313-320, 1993.

MIRANDA, H. S.; ROCHA E SILVA, E. P.; MIRANDA, A C. Comportamento do Fogo em Queimadas de Campo Sujo. In: MIRANDA, H. S.; DIAS, B. F. S.; SAITO, C. H. (Eds.) Impacto de queimadas em área de cerrado e restinga. Brasília: ECL/Universidade de Brasília, 1996. p. 1-10.

MOREIRA, A. G. Fire protection and vegetation dynamics in the brazilian Cerrado. 1992. $200 \mathrm{f}$. Tese (Ph.D.) - Harvard University, Harvard, 1992.

OLIVEIRA, R. S. et al. Efeito do fogo na floração de espécies de Orchidaceae em Cerrado. In: MIRANDA, H. S.; DIAS, B. F. S.; SAITO, C. H (Eds.) Impacto de queimadas em área de cerrado e restinga. Brasília: Universidade de Brasília: Brasília, 1996. p. 61-67.

OTTMAR, R. D. et al. Séries de estéreo-fotografias para quantificar a biomassa da vegetação do cerrado do Brasil Central. Washington:. USDA. 2001. (General Technical Report. PNW GTR 5191)

RAMOS, A. E. O efeito da queima sobre a vegetação lenhosa do cerrado. 1990. 142 f. Dissertação (Mestrado em Ecologia) - Universidade de Brasília, Brasília, 1990.

RAMOS-NETO, M. B. O Parque Nacional das Emas (GO) e o fogo: implicações para a conservação biológica. 2000. 159 f. Tese (Doutorado em Ecologia) - Universidade de São Paulo, São Paulo, 2000.

RATTER, J. A.; RIBEIRO, J. F.; BRIDGWATER, S. Woody flora distribution of the cerrado biome: phytogeography and conservation priorities. In: CAVALCANTI, T. B.; WALTER, B. M. T. (Eds.) Tópicos atuais em botânica. Brasília: Sociedade Botânica do Brasil/ Embrapa Recursos Genéticos e Biotecnologia. Brasília, 2000. p. 340-342.

REZENDE, A. V. Diversidade, estrutura, dinâmica e prognose do crescimento de um cerrado sensu stricto submetido a diferentes distúrbios por desmatamento. 2002. 243 f. Tese (Doutorado) - Universidade Federal do Paraná, Curitiba, 2002.

SAMBUICHI, R. H. R. Efeitos de longo prazo do fogo periódico sobre a fitossociologia da camada lenhosa de um cerrado em Brasília, DF. 1991. 144 f. Dissertação (Mestrado em Ecologia) - Universidade de Brasília, Brasília, 1991.

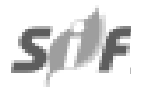

R. Árvore, Viçosa-MG, v.28, p.129-138, 2004 
SATO, M. N. Mortalidade de plantas lenhosas do cerrado submetidas a diferentes regimes de queima. 1996. 46 f. Dissertação (Mestrado em Ecologia) Universidade de Brasília, Brasília, 1996.

SILVA, E. P. R. Efeito do regime de queima na taxa de mortalidade e estrutura da vegetação lenhosa de campo sujo de Cerrado. 1999. 58 f. Dissertação (Mestrado em Ecologia) - Universidade de Brasília, Brasília, 1999.

SILVA, G. T.; SATO, M. N.; MIRANDA, H. S.

Mortalidade de plantas lenhosas em um campo sujo de cerrado submetido a queimas prescritas. In: MIRANDA, $\mathrm{H}$. S.; DIAS, B. F. S.; SAITO, C. H (Eds.) Impacto de queimadas em área de cerrado e restinga. Brasília: ECL/ Universidade de Brasília, 1996. p. 93-101.
SILVA Jr., M. C.; BATES, J. M. Biogeographic patterns and conservation in South American Cerrado: a tropical savanna hotspot. Bioscience, v. 52, n. 3, p. 225 233, 2002.

SOUZA, M. H. A. O.; SOARES, J. J. Brotamento de espécies arbustivas e arbóreas posteriormente a uma queimada, num Cerradão. In: SEMINÁRIO REGIONAL DE ECOLOGIA. 3., 1993. Brasília. Anais... Brasília: 1983. p. 263-275.

VIEIRA, E. M.; ANDRADE, I.; PRICE, P. W. Fire effects on a Palicourea rigida (Rubiaceae) gall midge: a test of the plant vigor hypothesis. Biotropica, v. 28, n. 2, p. 210-217, 1996. 